\title{
Refractory Head and Neck Carcinoma
}

National Cancer Institute

\section{Source}

National Cancer Institute. Refractory Head and Neck Carcinoma. NCI Thesaurus. Code C151907.

A head and neck carcinoma that does not respond to treatment. 